\title{
Establishment and Comparison of an Experimental Kidney Yang Deficiency Syndrome Rat Model Caused by Hydrocortisone, Adenine, and Dexamethasone Combined with Adriamycin in Rats
}

\author{
Yehui Zhang \\ Zhejiang Chinese Medical University \\ Junfeng Tong \\ Hangzhou yuanzhi medical technology sevice company \\ Yan Zhu \\ Zhejiang University School of Medicine First Affiliated Hospital \\ Qihan Luo \\ Zhejiang Chinese Medical University \\ Hongxia Yu \\ Zhejiang Chinese Medical University \\ Shuo Huang \\ Zhejiang Chinese Medical University \\ Liting Ji \\ Zhejiang Chinese Medical University \\ Changyu Li ( $\sim$ lcyzcmu@sina.com) \\ Zhejiang Chinese Medical University https://orcid.org/0000-0001-8656-0428
}

\section{Research}

Keywords: Kidney yang deficiency, Hypothalamic-pituitary-adrenal axis, Renal toxicity

Posted Date: December 2nd, 2020

DOI: https://doi.org/10.21203/rs.3.rs-117500/v1

License: (c) (1) This work is licensed under a Creative Commons Attribution 4.0 International License. Read Full License 


\section{Abstract}

Objective: The paper is aimed to establish a method for renal injury in rats with kidney yang deficiency syndrome (KYDS) of the stable by comparing different model establishment methods and optimize the final modeling method.

Method: In order to mimic water metabolism disorder of KYDS- a diagnostic pattern in traditional Chinese medicine (TCM), we made a comparison of hydrocortisone-induced, adenine-induced, dexamethasone combined with adriamycin-induced KYDS rat model according to testing general behavior, detecting Hypothalamus-pituitary-adrenal (HPA) axis function and renal function also making use of drug action. Additionally, the effect of adenine at a dose of $100 \mathrm{mg} / \mathrm{kg}, 150 \mathrm{mg} / \mathrm{kg}$, and $200 \mathrm{mg} / \mathrm{kg}$, respectively, on establishment of KYDS model was investigated in rats. Finally, Shenqi Wan (SQW, $3 \mathrm{~g} / \mathrm{kg}$ ) was used to estimate the deterioration of adenine that is associated with KYDS.

Result: Three KYDS model rats all exhibited weight loss, anal temperature drop, reduced food intake and dysfunction of the Hypothalamus-pituitary-adrenal (HPA) axis, which could be alleviated by SQW. However, different from other two methods, adenine-induced rats were prominently manifested as water and fluid metabolism disorders (sharply increased water intake and urine volume). Renal injury in adenine-induced rats was more obvious than it in hydrocortisone-induced rats and dexamethasone combined with adriamycin-induced rats. And renal toxicity caused by adenine was reversible by SQW treatment. Water metabolism disorder in adenine-induced rats was more closer to clinical symptoms of patients with KYDS. Subsequently, we found different dose of adenine gavage $(100,150,200 \mathrm{mg} / \mathrm{kg})$ for 14 days result in general behavior change, dysfunction of HPA axis and renal function damage. But only medium-dose of adenine induced toxicity could be reversed by SQW treatment for 21 days.

Conclusion: The result of the comparison between the three modeling methods showed that adenineinduced was more effective to establish the KYDS model, and only a medium-dose of adenine for inducing renal poison could be recovered by SQW treatment. Therefore, a medium-dose of adenine was optimal for the establishment of the KYDS rat model.

Trial registration : This is an animal experiment, trial registration is not necessary.

\section{Background}

Kidney yang deficiency syndrome (KYDS) is a diagnostic pattern in traditional Chinese medicine (TCM) that is related to multiple chronic diseases such as chronic renal failure, nephrotic syndrome, neuroimmune endocrine system dysfunction (1). KYDS is characterized by diarrhea, aversion cold, slow response, decreased appetite, and polyuria, etc (2). KYDS is associated closely with renal function damage and its important dialectical symptom is water metabolism disorder(3). And building a suitable animal model has become an important means for the scientific interpretation of the connotation of "the kidney governs water" in TCM. Modern medical research demonstrated that functional disorder of the HPA axis is the pathogenesis mechanism of KYDS, and many previous animal models of KYDS were 
constructed based on HPA axis disorders(4). However, these KYDS model animal cannot well mimic water-liquid metabolism disorder, this inhibits comprehensive exploration and research of KYDS.

Several animal models of KYDS have been applied in actual research, the most common one is animals given by high doses of adrenocorticotropic hormone such as hydrocortisone and dexamethasone (5-7). It was demonstrated that adenine deposits in the renal tubules, resulting in renal damage and developing into KYDS symptoms in rats (8-11). Recently, some experts put forward the idea of modeling basic diseases combined with multiple factors: firstly, inducing symptoms of KYDS in rats while maintaining the integrity of organs taking advantage of hydrocortisone, dexamethasone, and hydroxyurea. Secondly, rats are tail vein injected with adriamycin to bring about renal function loss $(4,12)$. Every method of establishing the KYDS animal model has its principle and leads to different physiological and pathological manifestations. However, how to filter out the optimal animal model of KYDS that better mimic water and fluid metabolism disorder in KYDS is urgent to be explored.

Evaluation of successful KYDS model establishment through tonifying kidney yang medicine is a necessary means (13). As a well-known prescription of treating KYDS, SQW is first recorded in Jin Kui Yao Lue written by Zhang Zhongjing, made up of Radix Rehmanniae Preparata, Poria, Rhizoma Alismatis, Rhizoma Dioscoreae Oppositae, Cortex Moutan Radicis, Fructus Macrocarpii, Achyranthes bidentata, and Plantagoasiatica (14) (15). Our previous research demonstrated that SQW could alleviate renal injury under KYDS through aquaporin 1(AQP1) (16). The progression of renal fibrosis could be slowed down by SQW through inhibiting Epithelial-mesenchymal transition (EMT) (13).

This study was aimed at making a comparison among three methods (Hydrocortisone, adenine, dexamethasone combined with adriamycin) to induce KYDS rat model. Renal function, neuroendocrine and water metabolism were evaluated to optimize the animal model of KYDS and make the model closer to the clinical manifestations of the water and fluid metabolism disorder of KYDS. Next, we optimize adenine dosage for establishing KYDS model.

\section{Materials And Methods}

\subsection{Rats}

A total of 256 Sprague Dawley rats aged 2-month-old and weighing 200-220 g were purchased from Shanghai Slake Laboratory Animal Co., LTD. (SCXK (Shanghai) 2012-0002). The rats were housed in a standard animal room, with food and water provided ad libitum in the Animal Experiment Research Center of Zhejiang University of Traditional Chinese Medicine (SYXK (Zhejiang) 2013 - 0184). The feeding conditions were as follows: constant temperature, temperature $22 \pm 1{ }^{\circ} \mathrm{C}$, humidity $50 \% \varangle 60 \%$, light and dark alternately every $12 \mathrm{~h}$, and the number of wind changes $15 \otimes 20$ times/h. All experiments of the animals were guided and approved by the Animal Care and Use Committee of Zhejiang Chinese medical university (Approval Number: ZSLL-2014-59).

\section{$2.2 \mathrm{SQW}$ preparation}


SQW (Henan Wanxi Pharmaceutical Co., Ltd, China, 111002) was dissolved in distilled water, prepared into a solution with a concentration of $0.3 \mathrm{~g} / \mathrm{mL}$ containing original medicinal materials according to our previous data (13). The clinical equivalent dose of SQW was calculated according to the conversion method of the dosage of experimental animals and humans (calculated by the ratio of the body surface area of $200 \mathrm{~g}$ rats with standard weight and $70 \mathrm{~kg}$ humans). According to the clinical dosage of this formula, the dosage of each administration group is equivalent to 3 times the clinical dosage.

\subsection{KYDS rat modeling protocol}

Sprague Dawley rats were randomly divided into seven groups (a control group, a hydrocortisone induced model group, an adenine induced model group, dexamethasone combined with adriamycin induced model group, a hydrocortisone + SQW group, an adenine + SQW group, dexamethasone combined with adriamycin + SQW group; $n=10$ per group). To establish the KYDS rat model, hydrocortisone induced model group and hydrocortisone + SQW group were intramuscular injected with $25 \mathrm{mg} / \mathrm{kg}$ hydrocortisone (Shanxi Jinxin Shuanghe Pharmaceutical Co., Ltd, China, 201009055) for 20 days, adenine induced model group and adenine + SQW group were administrated with $200 \mathrm{mg} / \mathrm{kg}$ adenine (Shanghai Bo'ao Biotechnology Co., Ltd, China, 120110) by oral gavage for 20 days, and dexamethasone (Chenxin Pharmaceutical Co., Ltd, China, 120119612) combined with adriamycin (Actacis Italy S.p.A, Italy, 0QL0064) induced model group and dexamethasone combined with adriamycin + SQW group were intramuscular injected with $1.8 \mathrm{mg} / \mathrm{kg}$ dexamethasone for 10 days and tail vein injected with $4 \mathrm{mg} / \mathrm{kg}$ adriamycin at the 11th day. The frequency of administration was once a day. Then from the 12th day of this experiment, $3 \mathrm{~g} / \mathrm{kg} \mathrm{SQW}$ was administrated by oral gavage into hydrocortisone + SQW group, adenine + SQW group, dexamethasone combined with adriamycin + SQW group for 14 days, while other groups treated with equal distilled water.

\subsection{Adenine poisoning protocol}

Sprague Dawley rats were randomly divided into 7 groups (a control group, a low-dose adenine group, a medium-dose adenine group and a high-dose adenine group, a low-dose adenine + SQW group, a medium-dose adenine + SQW group and a high-dose adenine + SQW group, $\mathrm{n}=30$ per group) according to body weight. The rats other than control group were administrated with adenine of respective 100,150 and $200 \mathrm{mg} / \mathrm{kg}$ by oral gavage once a day. 0.5\% CMC-Na was orally administrated into the control group equally. On the 7th day and 14th day, the establishment of the KYDS rat model was evaluated judging from the general behavior test, HPA axis, and renal function analysis. The kidney tissue was collected for $\mathrm{H} \& \mathrm{E}$ and Masson staining.

Once the KYDS rat model was successfully built, the low-dose adenine + SQW group (100 mg/kg), medium-dose adenine + SQW group $(150 \mathrm{mg} / \mathrm{kg})$, and high-dose adenine + SQW group $(200 \mathrm{mg} / \mathrm{kg})$ was treated with $3 \mathrm{~g} / \mathrm{kg}$ SQW while concurrently poisoned with a respective dose of adenine by oral gavage for continuous 21 days. The recovery effectS of SQW in each model rats were evaluated to determine the optimal dose of adenine.

\subsection{H\&E and Masson staining of rat renal tissue}


3 Sprague Dawley rats in each group were sacrificed every due time. The renal tissues were fixed with $10 \%$ formaldehyde, embedded in paraffin, and cut into 3-5 $\mu \mathrm{m}$ sections, and subjected to H\&E and Masson staining respectively. The pathological condition of the kidney tissue was observed under a 200X optical microscope. The results of H\&E staining were used to observe the basic morphology of rat kidney tissue and the results of Masson staining were used to evaluate the renal tubulointerstitial lesions according to the semi-quantitative scoring system. Each Masson stained section is randomly selected 10 fields of view under a 200 -fold light microscope. The blue is the fibrosis positive staining area. The ratio of the fibrosis area to the total area of the statistical field (excluding the glomeruli and large blood vessels) is used as the renal tubules Fibrosis index, to observe the degree of tubular interstitial fibrosis. The evaluation criteria are mild damage (fibrosis index $<25 \%$ ), moderate damage (fibrosis index $25 \%-50 \%$ ), severe damage (fibrosis index $>50 \%$ ), using color image analysis system (Image-Pro Plus, IPP) count the positive area.

2.6 Measurement of hypothalamus corticotrophin-releasing factor $(\mathrm{CRH})$, serum adrenocorticotrophic hormone (ACTH), cortisol (CORT), blood urea nitrogen (BUN) and serum creatinine (Scr)

$3 \%$ sodium pentobarbital solution was injected intraperitoneally to anesthetize the rats. Blood was collected from the ventricle and placed in a centrifuge tube. The blood was centrifuged at $3000 \mathrm{rpm}$ for 10 min $\left(4^{\circ} \mathrm{C}\right)$. The serum was separated, packed in a centrifuge tube, and stored at $-20^{\circ} \mathrm{C}$ for later use. Take the hypothalamus stored in liquid nitrogen, weigh it, place it in a $5 \mathrm{~mL}$ centrifuge tube, and add 9 times the volume of PBS homogenization buffer $(8.0 \mathrm{~g} \mathrm{NaCl}, 0.2 \mathrm{~g} \mathrm{KCl}, 1.44 \mathrm{~g} \mathrm{Na} 2 \mathrm{HPO} 4,0.24 \mathrm{~g} \mathrm{KH} 2 \mathrm{PO} 4$, add distilled water to $1000 \mathrm{~mL}$, Adjust the $\mathrm{pH}$ to 7.4), homogenize at high speed, and centrifuge for $20 \mathrm{~min}(3000 \mathrm{rpm})$. Take the supernatant and place it in a centrifuge tube and store it in a refrigerator at $-20{ }^{\circ} \mathrm{C}$ for later use. The level of hypothalamus $\mathrm{CRH}$, serum ACTH, CORT was measured by Elisa kit (Shanghai Xinfan Biotechnology, Shanghai, China). The level of serum BUN, Scr was evaluated by the automatic biochemical detector (7020, Hitachi Co., Ltd., Japan).

\subsection{Measurement of urinary 17-hydroxy corticosteroid (17- $\mathrm{OHCS}$ ) and 24-hour urine protein excretion (U-TP)}

$24 \mathrm{~h}$ urine of rats was centrifuged at $2000 \mathrm{rpm}$ for $5 \mathrm{~min}\left(4^{\circ} \mathrm{C}\right)$, collected and stored at $-20^{\circ} \mathrm{C}$. Elisa kit (Shanghai Xinfan Biotechnology, Shanghai, China) was used to measure the level of urine 17-OHCS. The automatic biochemical detector was performed to measure the level of U-TP.

\subsection{Analysis of AQP1 and AQP2 in renal tissue by Elisa kit}

The renal medulla was taken and weighted from renal tissue preserved in liquid nitrogen, the same preparation of the homogenate as mentioned before were followed. The level of AQP1 and AQP2 was analyzed by the ELISA kit (Shanghai Xinfan Biotechnology, Shanghai, China).

\subsection{Statistical analysis}


The SPSS17.0 statistical software is used to process the experimental data. The experimental data are expressed as Mean \pm standard deviation ( $x \pm s d$ ). Each group of data is tested for normality. The comparison of multiple samples means conforming to the normal distribution is analyzed by one-way ANOVA. If the variances are uniform, the LSD test is used, if the variance is not uniform, the GamesHowell $(A)$ test is used, $p<0.05$ means the difference is statistically significant; $p<0.01$ means the difference is extremely significant.

\section{Result}

3.1 Effect of different methods for establishing KYDS rat model on body weight, anal temperature, food intake, water intake, and urine volume

We examined the effect of hydrocortisone intravenous injection, adenine oral gavage, and dexamethasone combined with adriamycin injection on general behavior in rats and verify the optimal method of the KYDS model according to the evaluation of their response to SQW treatment. We found three modeling methods produced an obvious drop in body weight of rats compared with the control group $(P<0.01)$, which could not be elevated significantly by SQW (Fig. 1A). As shown in Fig. 1B that anal temperature in the HYD group, ADE group, and DEX + ADR group was hugely lower than in the CON group $(P<0.01)$. SQW treatment inhibited declining anal temperature respectively contrasted by HYD group $(P<$ $0.05)$, ADE group $(P<0.01)$, and $D E X+A D R$ group $(P<0.01)$. Compared with the $C O N$ group, reduced food intake was observed in HYD $(P<0.01), A D E(P<0.01)$, DEX + ADR $(P<0.01)$ group and attenuated by SQW (Fig. 1C). Urine volume of the HYD group was slightly increased compared with the CON group $(\mathrm{P}<$ $0.05)$, but only adenine gavage induced a sharp rise in water intake $(P<0.01)$ and urine volume $(P<0.01)$, both of which could be alleviated by SQW $(P<0.01, P<0.01)$ (Fig. 1D-E). These findings indicated that all three modeling methods exhibited body weight loss, anal temperature decline, food intake dropout which could be alleviated by SQW treatment, but only adenine gavage-induced rats had obvious water-liquid metabolism disorder, as evident by sharply increased water intake and urine volume.

3.2 Effect of different methods for establishing KYDS rat model on HPA axis function and renal function

It was observed in Fig. 2, all modeling methods led to the dysfunction of the HPA axis, as evident by obviously reduced hypothalamic $C R H(P<0.01)$, serum ACTH $(P<0.01)$, CORT $(P<0.01)$, and urine 17OHCS $(P<0.01)$ (Fig. 2A-D). SQW treatment elevated indicators of the HPA axis in KYDS rats (HYD, ADE, $D E X+A D R ; P<0.01$ for all). These results indicated that there was no obvious difference in HPA function inhibited by three modeling methods.

Dexamethasone combined with adriamycin significantly increased U-TP which was reduced by SQW treatment with no significant difference. However, BUN, Scr, and U-TP were all markedly increased in the $A D E$ group versus the control group, and SQW significantly decreased BUN in the ADE group and reduced Scr and U-TP but no significant difference (Fig. 2E-G). This result suggested reversible renal function damage of rats would be caused by adenine gavage. AQP1 and AQP2 is water channel protein reflecting water metabolism. Compared with the control group, AQP1 and AQP2 levels in kidney tissue were 
obviously reduced both in HYD and ADE model rats. It was analyzed by Elisa assay that the AQP2 level in the HYD + SQW group was markedly elevated contrasted by the HYD group, but there was no obvious difference of AQP2 level between the HYD group and HYD + SQW group. However, SQW supplementation successfully attenuated AQP1 and AQP2 levels in the kidney suppressed by adenine. As is shown in Fig. $2 \mathrm{H}-\mathrm{I}, 3 \mathrm{~g} / \mathrm{kg}$ SQW had a promotional effect on AQP1 and AQP2 level in the kidney inhibited by adenine but not hydrocortisone.

\subsection{Effect of different methods for establishing KYDS rat model on renal pathological change}

We observed clear glomerulus structure and normal tubules and interstitial substances in the CON group by light microscope in Fig. 3. In the HYD group, the size of the glomerulus increased slightly, the width of the mesangial area increased. In the ADE group, we found that the internal structure of the glomerulus collapsed, and the kidney glomerular fibrosis, renal tubule dilatation, interstitial inflammatory changes, adenine crystals. In the DEX + ADR group, glomerular size and width of the mesangial area were increased significantly as same as a renal pathological change in the HYD group. However, a more obvious pathological change was that eosinophilic red stains were exuded into a typical protein cast in the renal tubules. After SQW treatment, the renal pathology of each model group was alleviated.

Therefore, the result confirmed that adenine induced rats exhibited obvious water metabolism dysfunction and renal injury.

3.4 Effect of different dose of adenine on body weight, temperature, grip, food and water intake, urine volume in rats

As is shown in Fig. 4, compared with the normal control group, the body weight of $100 \mathrm{mg} / \mathrm{kg}$ adenineinduced rats was decreased but no significant difference while $150 \mathrm{mg} / \mathrm{kg}$ and $200 \mathrm{mg} / \mathrm{kg}$ adenineinduced rats had significant weight loss $(P<0.01$ ) (Fig. 4A). Meanwhile, model rats of $100 \mathrm{mg} / \mathrm{kg}$, $150 \mathrm{mg} / \mathrm{kg}$, and $200 \mathrm{mg} / \mathrm{kg}$ adenine had significantly lower temperatures than normal control rats $(\mathrm{p}<$ 0.01) (Fig. 4B). Compared with the normal control group, $100 \mathrm{mg} / \mathrm{kg}$ adenine group was obviously decreased in grip $(P<0.05)$ while the grip of $150 \mathrm{mg} / \mathrm{kg}$ adenine group and $200 \mathrm{mg} / \mathrm{kg}$ adenine group were decreased more sharply $(P<0.01)$ (Fig. $4 \mathrm{C})$. No remarkable food intake change was observed in $100 \mathrm{mg} / \mathrm{kg}$ and $150 \mathrm{mg} / \mathrm{kg}$ adenine-induced rats contrasted by the control group. Compared with the normal control group, the food intake of $200 \mathrm{mg} / \mathrm{kg}$ adenine group was significantly reduced $(P<0.01)$ (Fig. 4D). Water intake and urine volume of rats showed a sharp rise due to the different doses of adenine administration compared with the normal control group $(\mathrm{P}<0.01)$ (Fig. 4E-F). These findings indicated that $100 \mathrm{mg} / \mathrm{kg}, 150 \mathrm{mg} / \mathrm{kg}$ and $200 \mathrm{mg} / \mathrm{kg}$ adenine could damage the general physiological signs of rats.

\subsection{Effect of different dose of adenine on the hypothalamus-pituitary-adrenal (HPA) axis in rats}


HPA axis function is an important indicator for judge the successful construction of the KYDS model. As is shown in Fig. $5,100 \mathrm{mg} / \mathrm{kg}, 150 \mathrm{mg} / \mathrm{kg}$ and $200 \mathrm{mg} / \mathrm{kg}$ adenine administration for constant 7 days cannot cause damage of HPA axis function. However, three doses of adenine gavage for 14 days significantly decreased serum ACTH compared with the control group $(P<0.05, P<0.05, P<0.01$ respectively) (Fig. 5A). And model rats induced by $150 \mathrm{mg} / \mathrm{kg}$ and $200 \mathrm{mg} / \mathrm{kg}$ adenine for constant 14 days had markedly decreased in serum CORT $(P<0.01)$ and urine 17-OHCS $(P<0.01)$ contrasted by the control group (Fig. 5B-C). These findings indicated that adenine administration for 7 days was not suitable for KYDS model establishment, thereby we mainly focus on the effect of adenine administration for 14 days. We observed the effect of adenine administration for 14 days on the HPA axis function of rats was dose-dependent.

\subsection{Effect of different dose of adenine on renal function in rats}

It was observed in Fig. 5 that after 7 day adenine administration, $B U N(P<0.01)$ and $\operatorname{Scr}(P<0.01)$ were significantly increased in all model group (ADE $100 \mathrm{mg} / \mathrm{kg}$, ADE $150 \mathrm{mg} / \mathrm{kg}$, ADE $200 \mathrm{mg} / \mathrm{kg}$ ) versus control group (CON) and $100 \mathrm{mg} / \mathrm{kg}$ and $200 \mathrm{mg} / \mathrm{kg}$ adenine induced rats (ADE $100 \mathrm{mg} / \mathrm{kg}$, ADE $200 \mathrm{mg} / \mathrm{kg}$ ) had significantly increased in U-TP $(\mathrm{P}<0.05, \mathrm{P}<0.01$ respectively) compared with control group (CON). However, compared with the control group (CON), BUN $(P<0.01), \operatorname{Scr}(P<0.01)$, and U-TP $(P$ $<0.01$ ) were elevated in rats treated with continuous 14 day adenine gavage (ADE $100 \mathrm{mg} / \mathrm{kg}, A D E$ $150 \mathrm{mg} / \mathrm{kg}$, ADE $200 \mathrm{mg} / \mathrm{kg}$ ) (Fig. 5D-F). These findings suggested that there was a dose-dependent effect of adenine administration for 14 days on renal function in rats.

\subsection{Renal pathology of rats induced by the different dose of adenine}

According to the H\&E staining result of kidney tissue, we observed normal glomerulus morphology, clear tubular interstitial structure, normal cortical, and medullary structure in the CON group. Adenine gavage led to the increased size of the glomerulus and the increasing width of the mesangial area (Fig. 6A). As is shown in Fig. 6B, Masson staining analysis indicated that both 7 day and 14 day-different doses of adenine gavage produced renal interstitial fibrosis. Compared with the CON group, the percent of Masson positive area in each model group was evidently elevated $(P<0.01)$. These findings indicated that three doses of adenine led to renal interstitial fibrosis in rats with a dose-dependent effect.

3.8 Effect of SQW on body weight, temperature, grip, food and water intake, urine volume in KYDS rats induced by the different dose of adenine

As is shown in Fig. 7-8, SQW was used to test out optimal adenine dose, judging from which dose of adenine-induced renal toxicity was reversible. Compared with the CON group, body weight $(P<0.01)$, anal temperature $(P<0.01)$, grip $(P<0.01)$ were decreased and water intake $(P<0.05)$, urine volume $(P<0.01)$ were significantly increased but food intake was unstable in ADE $100 \mathrm{mg} / \mathrm{kg}$ group. Compared with $A D E$ 
$100 \mathrm{mg} / \mathrm{kg}$ group, SQW possessed a recovery effect on anal temperature $(P<0.01)$ and grip $(P<0.01)$ in ADE $100 \mathrm{mg} / \mathrm{kg}+\mathrm{SQW}$ group but had no protective effect on other makers. ADE $150 \mathrm{mg} / \mathrm{kg}$ group exhibited weight loss $(P<0.01)$, low anal temperature $(P<0.01)$, declined grip $(P<0.01)$, decreased food intake $(P<0.01)$ and high water intake $(P<0.01)$ and urine volume $(P<0.01)$, SQW played an

ameliorating role in $150 \mathrm{mg} / \mathrm{kg}$ adenine caused general behavior imbalance $(P<0.01$ for all). $200 \mathrm{mg} / \mathrm{kg}$ adenine could successfully maintain damage of general behavior in rats $(P<0.01)$, but the only anal temperature was significantly elevated in ADE $200 \mathrm{mg} / \mathrm{kg}+$ SQW group versus ADE $200 \mathrm{mg} / \mathrm{kg}$ group. These findings indicated three doses of adenine all resulted in damage of general behavior but only $150 \mathrm{mg} / \mathrm{kg}$ adenine-induced general behavior imbalance could be alleviated by SQW.

Figure 8 Recovery effect of SQW on food intake, water intake and $24 \mathrm{~h}$ urine volume in KYDS rats induced by the different doses of adenine. (A) effect of $100 \mathrm{mg} / \mathrm{kg} \mathrm{SQW}$ on food intake in different doses of adenine induced KYDS rats. (B) effect of $150 \mathrm{mg} / \mathrm{kg} \mathrm{SQW}$ on food intake in different doses of adenine induced KYDS rats. (C) effect of $200 \mathrm{mg} / \mathrm{kg} \mathrm{SQW}$ on food intake in different doses of adenine induced KYDS rats. (D) effect of $100 \mathrm{mg} / \mathrm{kg} \mathrm{SQW}$ on water intake in different doses of adenine induced KYDS rats. (E) effect of $150 \mathrm{mg} / \mathrm{kg} \mathrm{SQW}$ on water intake in different doses of adenine induced KYDS rats. (F) effect of $200 \mathrm{mg} / \mathrm{kg} \mathrm{SQW}$ on water intake in different doses of adenine induced KYDS rats. (G) effect of $100 \mathrm{mg} / \mathrm{kg}$ SQW on urine volume in different doses of adenine induced KYDS rats. (H) effect of $150 \mathrm{mg} / \mathrm{kg}$ SQW on urine volume in different doses of adenine induced KYDS rats. (I) effect of $200 \mathrm{mg} / \mathrm{kg}$ SQW on urine volume in different doses of adenine induced KYDS rats. $\mathrm{n}=10$. Small horizontal bars indicate the mean \pm s.d. $\Delta P<0.05, \Delta \Delta P<0.01$ compared with the CON group; ${ }^{*}<<0.05$, $\star \star P<0.01$ compared with the ADE group.

3.9 Effect of SQW on HPA axis function in KYDS rats induced by the different dose of adenine

As is shown in Fig. 9, serum ACTH, CORT, and urine 17-OHCS were obviously diminished in all model rats (ADE $100 \mathrm{mg} / \mathrm{kg}$, ADE $150 \mathrm{mg} / \mathrm{kg}$, ADE $200 \mathrm{mg} / \mathrm{kg}$ ) versus the control group (CON). There was no significant recovery effect of SQW on HPA function damage induced by $100 \mathrm{mg} / \mathrm{kg}$ and $200 \mathrm{mg} / \mathrm{kg}$ adenine. Only compared with ADE $150 \mathrm{mg} / \mathrm{kg}$ group, $3 \mathrm{~g} / \mathrm{kg} \mathrm{SQW}$ treatment could markedly increase serum CORT $(P<0.05)$ and urine 17-OHCS $(P<0.05)$ in rats, suggesting $150 \mathrm{mg} / \mathrm{kg}$ adenine caused dysfunction of HPA axis could be alleviated by $3 \mathrm{~g} / \mathrm{kg} \mathrm{SQW}$ (Fig. 9A-C).

3.10 Effect of SQW on renal function in KYDS rats induced by the different dose of adenine

As is shown in Fig. 9, BUN, Scr and U-TP were significantly elevated in all model rats (ADE $100 \mathrm{mg} / \mathrm{kg}$, ADE $150 \mathrm{mg} / \mathrm{kg}, A D E 200 \mathrm{mg} / \mathrm{kg}$ ) versus the control group (CON). SQW administration significantly diminished BUN $(P<0.01)$ in ADE $200 \mathrm{mg} / \mathrm{kg}$ group and $\operatorname{Scr}(P<0.01)$ in ADE $100 \mathrm{mg} / \mathrm{kg}$. However, $150 \mathrm{mg} / \mathrm{kg}$ adenine-induced renal function damage would be alleviated by SQW, as evident by markedly declined BUN, Scr, and U-TP level ( $P<0.01$ for all) (Fig. 9D-F).

3.11 Effect of SQW on renal pathology in KYDS rats induced by the different dose of adenine 
H\&E staining in Fig. 10A suggested that adenine gavage increased the size of the glomerulus and width of the mesangial area. And SQW treatment could ameliorate renal toxicity resulted from adenine. We analyzed Masson staining picture that the percent of Masson positive area in renal tissue in all model groups was elevated sharply compared with the CON group ( $P<0.01$ for all) (Fig. 10B). However, SQW had a recovery effect on $100 \mathrm{mg} / \mathrm{kg}(P<0.01)$ and $150 \mathrm{mg} / \mathrm{kg}(P<0.01)$ adenine-induced renal toxicity but was ineffective in $200 \mathrm{mg} / \mathrm{kg}$ adenine induced group.

\section{Discussion}

KYDS is a comprehensive manifestation of renal dysfunction caused by damage of "kidney yang" (17). KYDS is located in the kidney, hence, renal function analysis is an important indicator for measuring successful establishment of KYDS model from the perspective of pathology and the pathogenesis of Chinese medicine. The present study carried on establishing and comparison of three KYDS rat models and found that there were similarities and differences between them. In this study, it was agreed that the symptoms and manifestations of three methods induced rats were also well in line with the diagnosis of the syndrome of kidney-yang deficiency, that is, the weakness and cold appearance of the animals were similar to those of patients with "yang deficiency" (18). Modern research has indicated that damage to HPA function was major pathological mechanisms underlying KYDS (19). As expected, three methods induced rats had sharply declined hypothalamus $\mathrm{CRH}$, serum ACTH, and CORT, and urine 17-OHCS levels compared with control rats, suggesting all three methods successfully built KYDS model rats.

However, they were different in renal function and water balance. We observed that different from the other two methods, adenine induced rats exhibited high water intake and urine volume, damaged renal tubules and glomeruli structure found in H\&E staining of kidney tissue. These findings were similar to symptoms of water metabolism disorder in patients of KYDS. Otherwise, BUN, Scr and UTP were key makers reflecting renal function. Adenine led to renal function loss in rats, as evidenced by increased BUN, Scr, and UTP. However, hydrocortisone-induced, dexamethasone combined with adriamycin-induced rats unable to appear obvious water metabolism disorder. Currently, aquaporin (AQP) is closely related to water metabolism in the body. It is an important substance that has been discovered in the past decade to regulate the absorption, distribution, secretion, and excretion of various parts of the mammalian body(20). AQP is distributed in multiple organs and tissues throughout the body, and is specifically involved in cytological functions such as the transportation, absorption, secretion of body fluids, cell migration, fat metabolism, epidermal renewal, nerve signal transduction, volume regulation, etc. Each AQP has its specific tissue distribution, and different AQPs have different roles in the process of water absorption and secretion in the kidney and other organs (21). In the original urine produced in the anterior part of the renal tubule, $80 \%$ of the water is reabsorbed through AQP1 (22), and at the end of the renal tubule, $10 \%$ of the water is reabsorbed through AQP2 (23). In this study, AQP1 and AQP2 levels in rat kidney tissue were estimated to analyze water metabolism capacity. Our findings indicated hydrocortisone and adenine elevated AQP1 and AQP2 levels in kidney tissue. In general, adenine administration not only maintained HPA axis dysfunction in rats but also produced water metabolism disorder of KYDS. 
These findings were consistent with characteristics of three KYDS model. Hydrocortisone is an exogenous glucocorticoid. When high dose of exogenous glucocorticoid is taken in, the adrenocorticotropin secreted by the pituitary is inhibited, resulting in a decrease in the secretion of adrenal corticosteroids and atrophy of the adrenal glands, resulting in dysfunction of HPA axis. However, some people believe that this type of model is far from human "Yang deficiency". Patients with "Yang deficiency" have low cortical function, but this type of "Yang deficiency" model have significantly higher plasma cortisol. And hydrocortisone administration cannot cause obvious damage to kidney in rats, which was contrary to the facts. In addition, as a new modeling method of different drug combinations, dexamethasone combined with adriamycin induced KYDS model is lack of data support. Although dexamethasone combined with adriamycin induced rats showed mild kidney damage, but didn't undermine water balance such as normal water intake, urine volume, AQP1 and AQP2 level in the kidney. Compared with the above two modeling methods, adenine induced rats exhibited water metabolism disorder and reversible renal injury, and general signs of "Yang deficiency". The limitation is that adenine induced renal injury commonly belonged to CKD (One of the important syndromes of kidney yang deficiency) in terms of etiology. In summary, adenine induced rat model may be used as a good KYDS model to study the notion of "kidney governs water" in TCM.

Large dose of adenine is easy to cause excessive nephrotoxicity, which could be observed in this study. Therefore, to optimize the method for building adenine induced KYDS model, we made a comparison of different doses of adenine $(100 \mathrm{mg} / \mathrm{kg}, 150 \mathrm{mg} / \mathrm{kg}, 200 \mathrm{mg} / \mathrm{kg})$ induced renal toxicity, detecting that different doses of adenine administration all successfully established the KYDS model. But according to the reversible judgment of adenine-induced renal toxicity through SQW treatment, only constant $150 \mathrm{mg} / \mathrm{kg}$ adenine administration could maintain an excellent effect of renal toxicity which could be reversible by SQW treatment. Therefore, $150 \mathrm{mg} / \mathrm{kg}$ was the optimal dose to establish KYDS model.

\section{Conclusion}

In conclusion, the rats were administrated with adenine exhibited a significant disorder of HPA axis compared with rats injected with hydrocortisone and dexamethasone combined with adriamycin, respectively, suggesting that adenine-induced was more effective to establish the KYDS model. This approach will be useful for studying the pathology of KYDS model and for developing new therapies.

\section{Abbreviations}

ANOVA one-way analysis of variance

H\&E hematoxylin-eosin

TCM traditional Chinese medicine

HPA Hypothalamus-pituitary-adrenal 
KYDS kidney yang deficiency syndrome

SQW Shenqi Wan

EMT Epithelial-mesenchymal transition

$\mathrm{CRH}$ corticotrophin-releasing factor

ACTH adrenocorticotrophic hormone

CORT cortisol

BUN blood urea nitrogen

Scr serum creatinine

U-TP 24-hour urine protein excretion

17-OHCS 17-hydroxy corticosteroid

AQP1 Aquaporin 1

AQP2 Aquaporin 2

CMC-Na Carboxymethylcellulose Sodium

CKD Chronic kidney disease

\section{Declarations}

\section{Ethics approval and consent to participate}

The animal experiments were approved by the Animal Care and Use Committee of Zhejiang Chinese medical university (Approval Number: ZSLL-2014-59).

\section{Consent for publication}

This manuscript is approved by all authors for publication.

\section{Availability of data and materials}

All data of this study can be found within the manuscript and available from the corresponding author upon request.

\section{Competing interests}

The authors declare that they have no competing interests. 


\section{Authors' contributions}

JT and YZ conceptualized the study design; $Y Z$ analyzed the data and interpreted the results; $Y Z$ wrote the initial drafts of the manuscript; $C L$, $L J$ and $Y W$ revised the manuscript. All authors read and approved the final manuscript.

\section{Funding}

This study was supported by the National Natural Science Foundation of China (grant no.8167151544)

\section{Footnotes}

Publisher's Note

Springer Nature remains neutral with regard to jurisdictional claims in published maps and institutional affiliations.

\section{References}

1. Nan Y, Zhou X, Liu Q, Zhang A, Guan Y, Lin S, et al. Serum metabolomics strategy for understanding pharmacological effects of ShenQi pill acting on kidney yang deficiency syndrome. J Chromatogr B Analyt Technol Biomed Life Sci. 2016;1026:217-26.

2. Chen R, Wang J, Zhan R, Zhang L, Wang X. Fecal metabonomics combined with $16 \mathrm{~S}$ rRNA gene sequencing to analyze the changes of gut microbiota in rats with kidney-yang deficiency syndrome and the intervention effect of You-gui pill. J Ethnopharmacol. 2019;244:112139.

3. Yajing $\mathrm{H}$, Jiaxu C. Formation Mechanism of Polyuria and Oliguria in View of Kidney Yang Deficiency. J Tradit Chin Med. 2017;58(09):741-4.

4. Pengfei Z, Xinxue Z, Zongjiang Z. Study and Reflection on Animal Models of Kidney-yang Deficiency Syndrome in Chronic Kidney Disease. Modernization of Traditional Chinese Medicine and Materia Medica-World Science and Technology. 2020:6.

5. Gao J, Yu J, Xu P, Chen J, Gao G, Li B, et al. Established UPLC-MS/MS procedure for multicomponent quantitative analysis in rat plasma: A contrastive pharmacokinetics study of Qiangshen tablet in normal and kidney yang deficiency syndrome models. J Chromatogr B Analyt Technol Biomed Life Sci. 2019;1106-1107:35-42.

6. Tan Y, Liu X, Lu C, He X, Li J, Xiao C, et al. Metabolic profiling reveals therapeutic biomarkers of processed Aconitum carmichaeli Debx in treating hydrocortisone induced kidney-yang deficiency syndrome rats. J Ethnopharmacol. 2014;152(3):585-93.

7. Yang S, Xu X, Xu H, Xu S, Lin Q, Jia Z, et al. Purification, characterization and biological effect of reversing the kidney-yang deficiency of polysaccharides from semen cuscutae. Carbohydr Polym. 2017;175:249-56. 
8. Zhang JY, Hong CL, Chen HS, Zhou XJ, Zhang YJ, Efferth T, et al. Target Identification of Active Constituents of Shen Qi Wan to Treat Kidney Yang Deficiency Using Computational Target Fishing and Network Pharmacology. Front Pharmacol. 2019;10:650.

9. Diwan V, Brown L, Gobe GC. Adenine-induced chronic kidney disease in rats. Nephrology (Carlton). 2018;23(1):5-11.

10. de Frutos S, Luengo A, Garcia-Jerez A, Hatem-Vaquero M, Griera M, O'Valle F, et al. Chronic kidney disease induced by an adenine rich diet upregulates integrin linked kinase (ILK) and its depletion prevents the disease progression. Biochim Biophys Acta Mol Basis Dis. 2019;1865(6):1284-97.

11. Morishita $Y$, Ohnishi A, Watanabe $M$, Ishibashi K, Kusano E. Establishment of acute kidney injury mouse model by $0.75 \%$ adenine ingestion. Ren Fail. 2011;33(10):1013-8.

12. Yingying C, Jing L, Yuan X, Qingwen T. Evaluation and Progress of Modeling Method about Kidney Yang Deficiency Animal Model. Chinese Archives of Traditional Chinese Medicine. 2018(11):2697700.

13. Chen H, Xu Y, Yang Y, Zhou X, Dai S, Li C. Shenqiwan Ameliorates Renal Fibrosis in Rats by Inhibiting TGF-beta1/Smads Signaling Pathway. Evid Based Complement Alternat Med. 2017;2017:7187038.

14. Zhou X-H, Zhang A-H, Wang L, Tan Y-L, Guan Y, Han Y, et al. Novel chinmedomics strategy for discovering effective constituents from ShenQiWan acting on ShenYangXu syndrome. Chin J Nat Med. 2016;14(8):561-81.

15. Ji B, Li YY, Yang WJ, Zhang LZ, Fang MS, Fu HY, et al. Jinkui Shenqi Pills Ameliorate Asthma with "Kidney Yang Deficiency" by Enhancing the Function of the Hypothalamic-Pituitary-Adrenal Axis to Regulate T Helper 1/2 Imbalance. Evid Based Complement Alternat Med. 2018;2018:7253240.

16. He Y, Bao YT, Chen HS, Chen YT, Zhou XJ, Yang YX, et al. The Effect of Shen Qi Wan Medicated Serum on NRK-52E Cells Proliferation and Migration by Targeting Aquaporin 1 (AQP1). Med Sci Monit. 2020;26:e922943.

17. Wang X, Zhang A, Zhou X, Liu Q, Nan Y, Guan Y, et al. An integrated chinmedomics strategy for discovery of effective constituents from traditional herbal medicine. Sci Rep. 2016;6:18997.

18. Ling S, Xu JW. Model organisms and traditional chinese medicine syndrome models. Evid Based Complement Alternat Med. 2013;2013:761987.

19. Tang N, Liu L, Qiu H, Shi W, Mao D. Analysis of gene expression and functional changes of adrenal gland in a rat model of kidney yang deficiency syndrome treated with Sini decoction. Exp Ther Med. 2018;16(4):3107-15.

20. Verkman AS, Anderson MO, Papadopoulos MC. Aquaporins: important but elusive drug targets. Nat Rev Drug Discov. 2014;13(4):259-77.

21. He J, Yang B. Aquaporins in Renal Diseases. Int J Mol Sci. 2019;20(2).

22. Pallone TL, Edwards A, Ma T, Silldorff EP, Verkman AS. Requirement of aquaporin-1 for $\mathrm{NaCl}$-driven water transport across descending vasa recta. J Clin Invest. 2000;105(2):215-22. 
23. BL SN, EI S, MA C. K, P A. CHIP28 water channels are localized in constitutively water-permeable segments of the nephron. J Cell Biol. 1993;120(2):371-83.

\section{Figures}
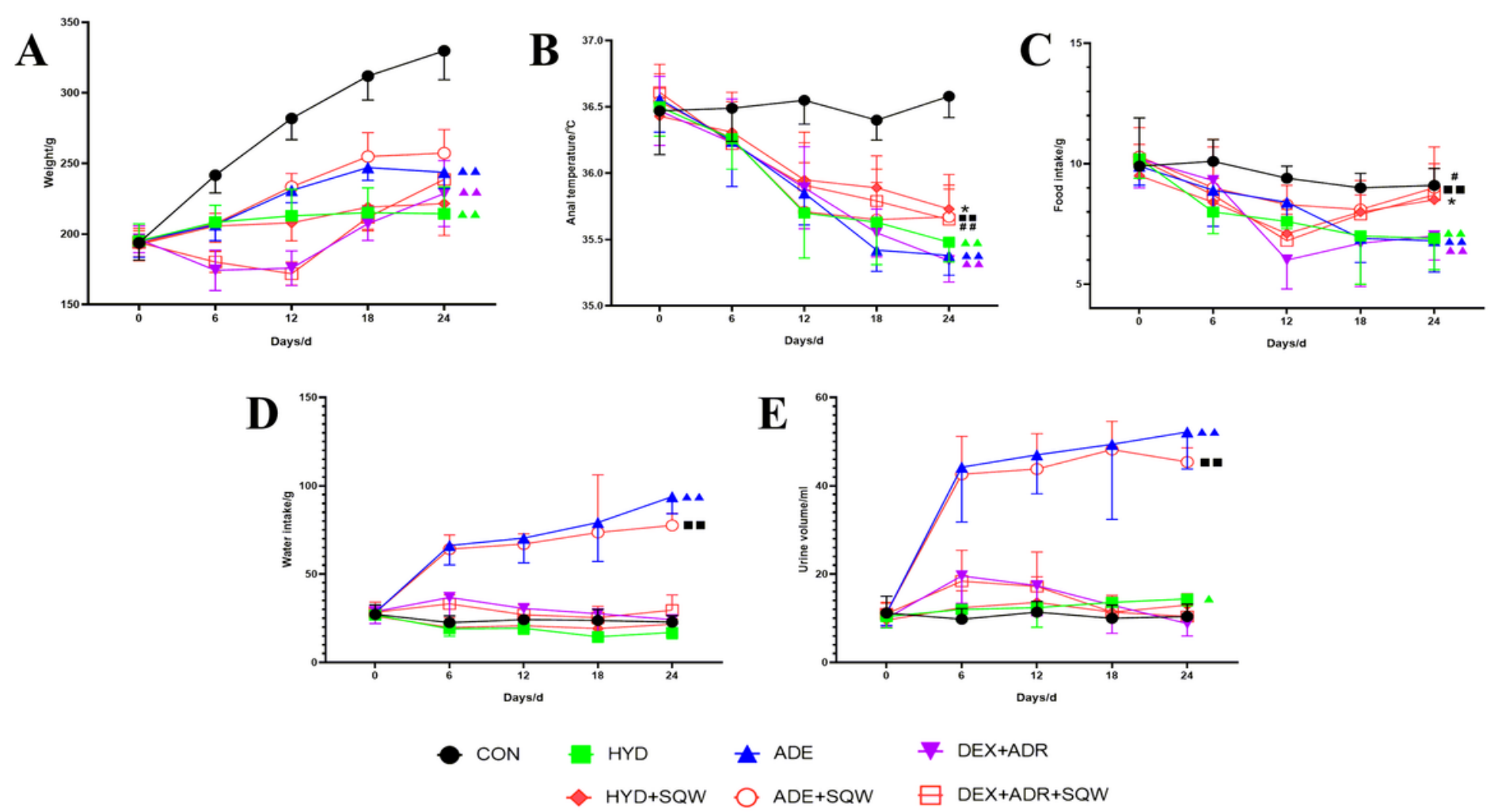

^ $A D E$
ADE+SQW

$\checkmark \mathrm{DEX}+\mathrm{ADR}$

$\boxminus D E X+A D R+S Q W$

\section{Figure 1}

Effect of different methods of establishing the KYDS rat model on body weight, anal temperature, food intake, water intake and $24 \mathrm{~h}$ urine volume. $n=10$. Small horizontal bars indicate the mean $\pm s$. $d$. (A) body weight change of three KYDS model rats and the effect of SQW. (B) anal temperature change of three KYDS model rats and the effect of SQW. (C) food intake change of three KYDS model rats and the effect of SQW. (D) water intake change of three KYDS model rats and the effect of SQW. (E) urine volume change of three KYDS model rats and effect of SQW. $\mathbf{\Delta} P<0.05, \boldsymbol{\Delta} \mathbf{\Delta} P<0.01$ compared with the CON group; ${ }^{*} P<0.05$, ${ }^{* *} P<0.01$ compared with the HYD group. $\square<0.05$, $\square P<0.01$ compared with the ADE group; $\# \mathrm{P}<0.05$, \# \#P<0.01 compared with the DEX+ADR group. (CON: control group, HYD: hydrocortisone induced group, $A D E$ : adenine induced group, $D E X+A D R$ : dexamethasone combined with adriamycin induced group, HYD+SQW: hydrocortisone induced group treated with SQW, ADE+SQW: adenine induced 

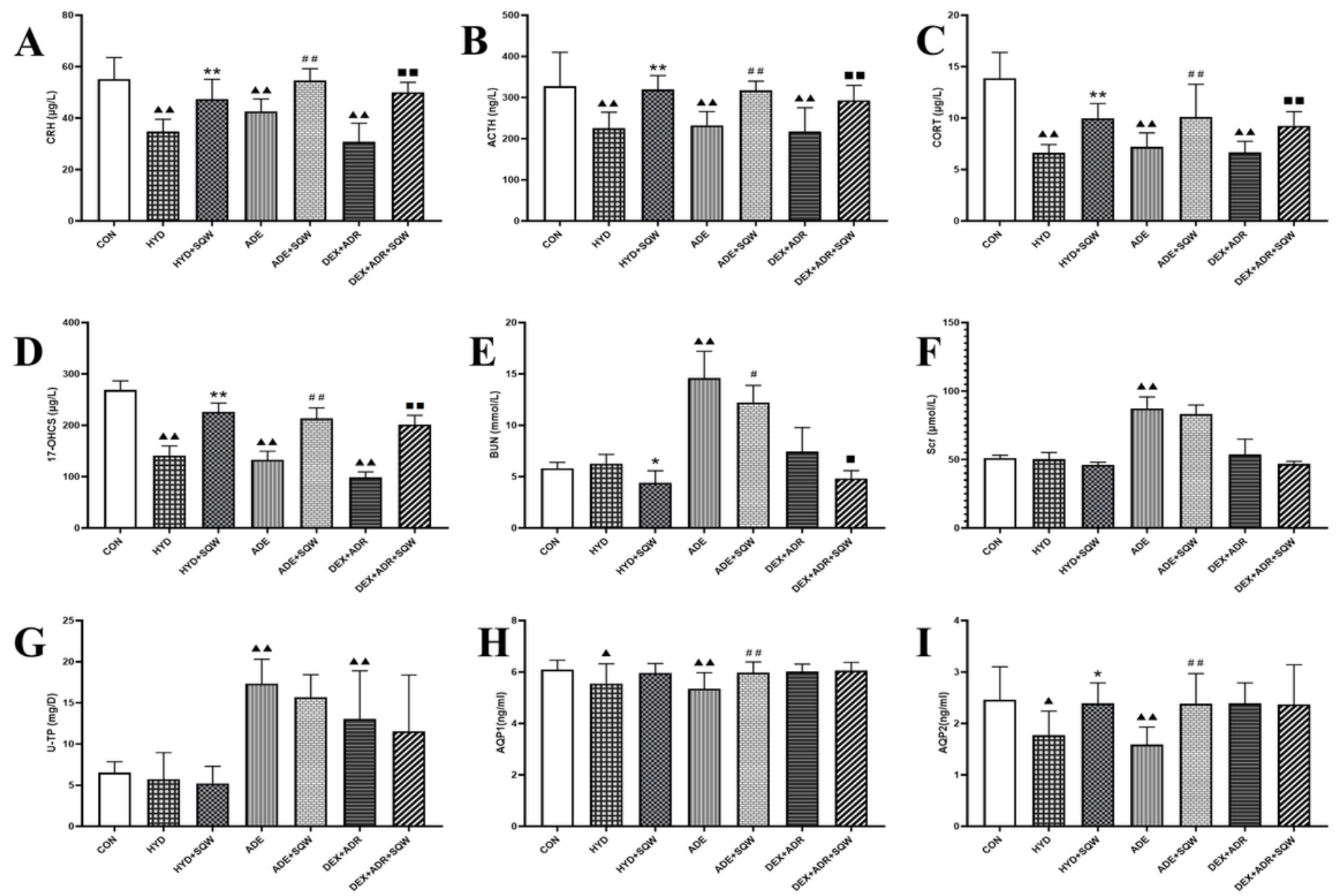

Figure 2

Effect of different methods of establishing the KYDS rat model on HPA axis function and renal function. (A-D) indices for HPA axis function, including levels of hypothalamic $\mathrm{CRH}$, serum ACTH, CORT, and urine 17-OHCS in rats. (E-G) indices for renal function, including levels of blood urea nitrogen (BUN), serum creatinine (Scr), 24-hour urine protein excretion (U-TP) in rats. (H-I) Analysis of AQP1 and AQP2 expression level in the kidney of rats by Elisa assay. $n=10$. Small horizontal bars indicate the mean \pm s.d. $\Delta P<0.05, \Delta \Delta P<0.01$ compared with the CON group; ${ }^{*} P<0.05$, ${ }^{* *} P<0.01$ compared with the HYD group. \#P<0.05, \# \#P<0.01 compared with the ADE group; $\square \mathrm{P}<0.05$, $\square \mathrm{P}<0.01$ compared with the DEX+ADR group. 


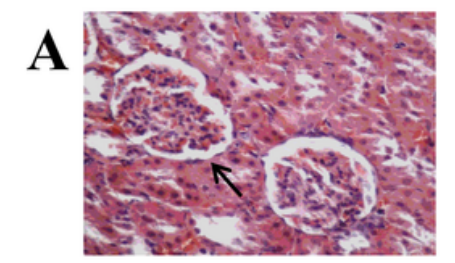

$\mathrm{CON}$

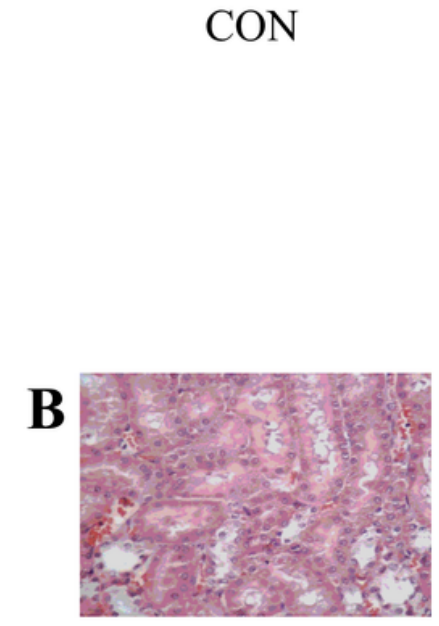

$\mathrm{CON}$

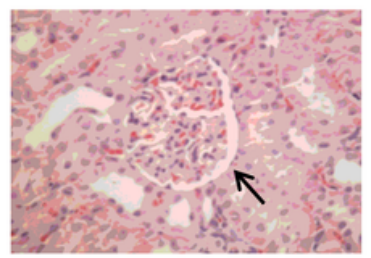

HYD

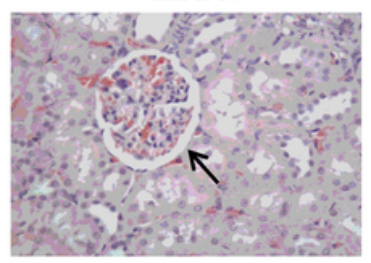

HYD+SQW

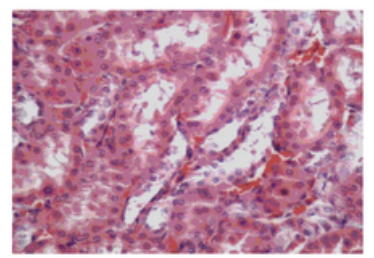

HYD

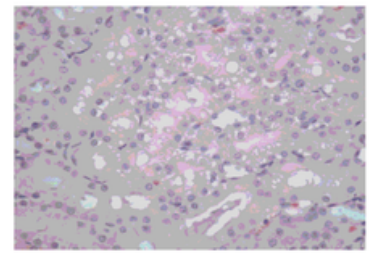

HYD+SQW

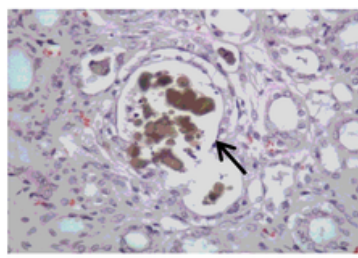

ADE

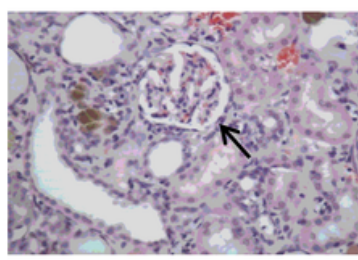

$\mathrm{ADE}+\mathrm{SQW}$

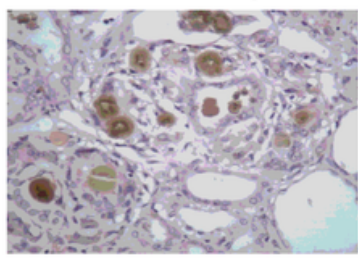

ADE

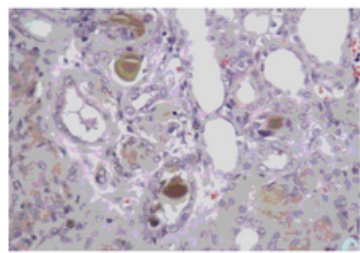

$\mathrm{ADE}+\mathrm{SQW}$

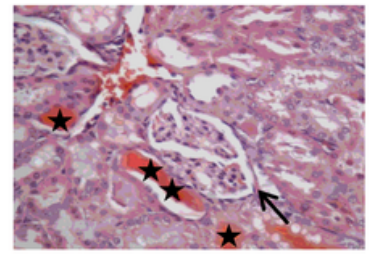

$\mathrm{DEX}+\mathrm{ADR}$

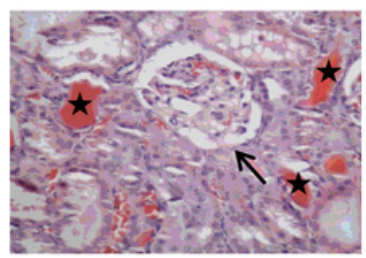

$\mathrm{DEX}+\mathrm{ADR}+\mathrm{SQW}$

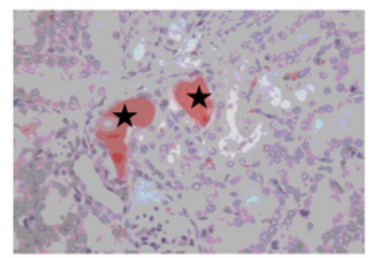

DEX+ADR

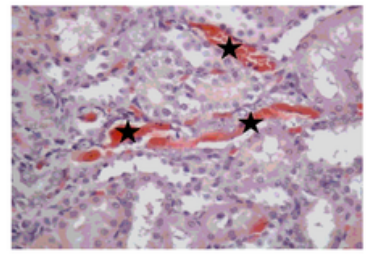

$\mathrm{DEX}+\mathrm{ADR}+\mathrm{SQW}$

\section{Figure 3}

Effect of different methods of establishing the KYDS rat model on renal pathological change. (A) Respective images of HE stained glomerulus in rats at a magnification of 400X. (B) Respective images of HE stained kidney tubules in rats at a magnification of $400 X . n=10$. Small horizontal bars indicate the mean \pm s.d. 

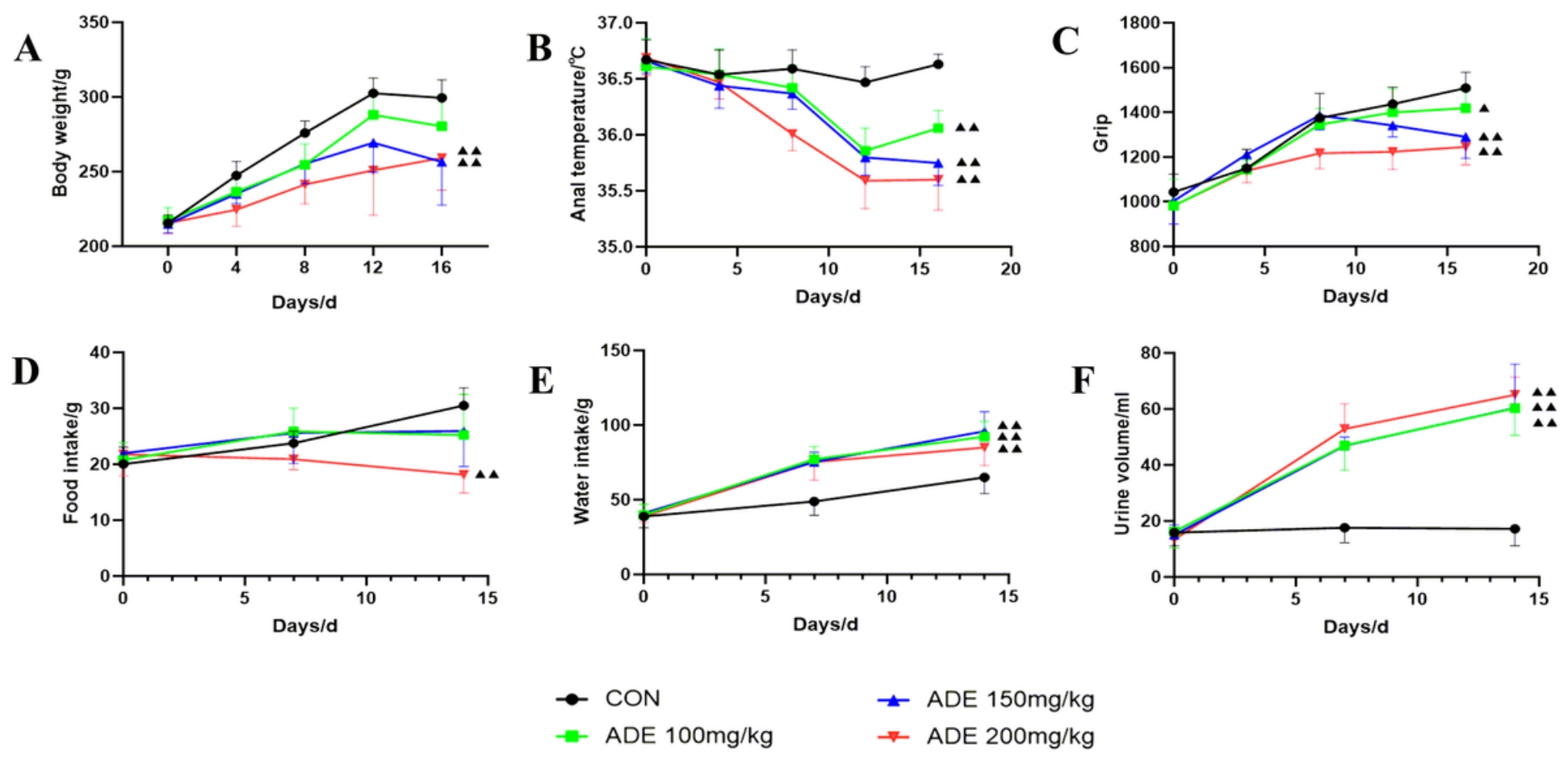

\section{Figure 4}

Effect of different doses of adenine on body weight, anal temperature, grip, food intake, water intake, and $24 \mathrm{~h}$ urine volume in rats. $(\mathrm{A})$ body weight change in rats induced by different doses of adenine. (B) anal temperature change in rats induced by different doses of adenine. (C) grip change in rats induced by different doses of adenine. (D) food intake change in rats induced by different doses of adenine. (E) water intake change in rats induced by different doses of adenine. $(F)$ urine volume change in rats induced by different doses of adenine. $n=10$. Small horizontal bars indicate the mean \pm s.d. $\Delta P<0.05$, $\Delta \mathbf{\Delta} P<0.01$ compared with the CON group. 
A
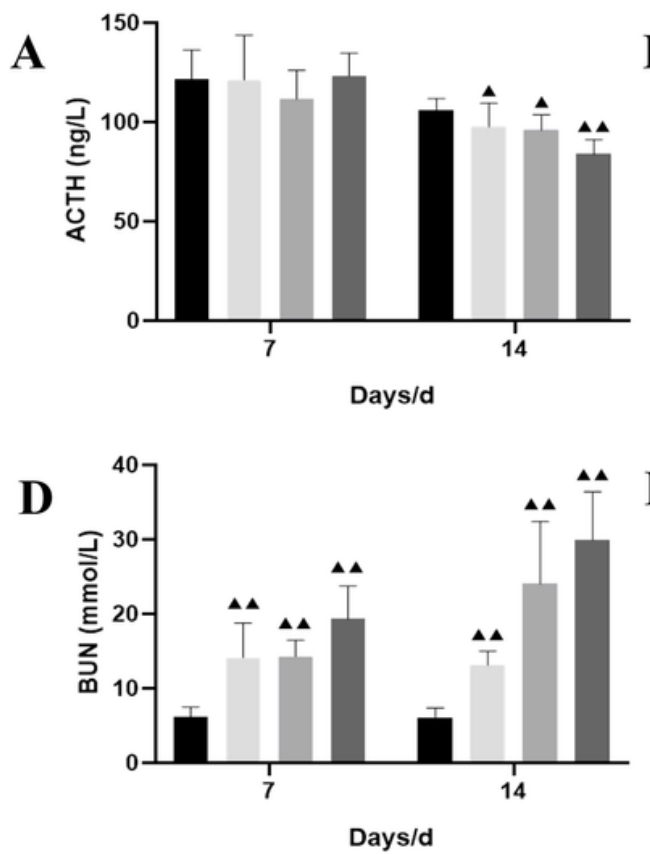

B

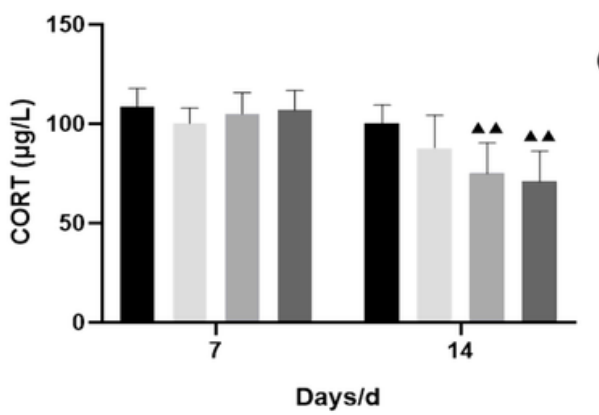

$\mathbf{E}$

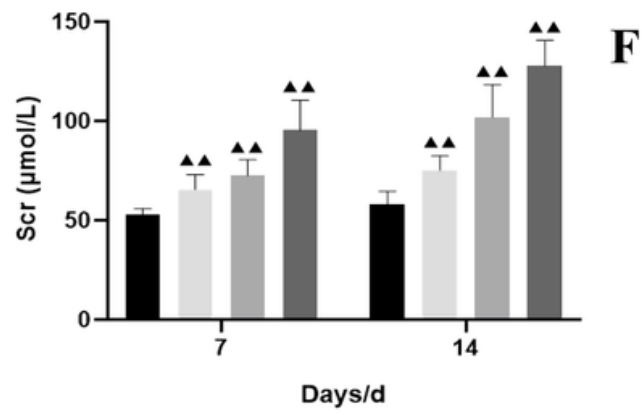

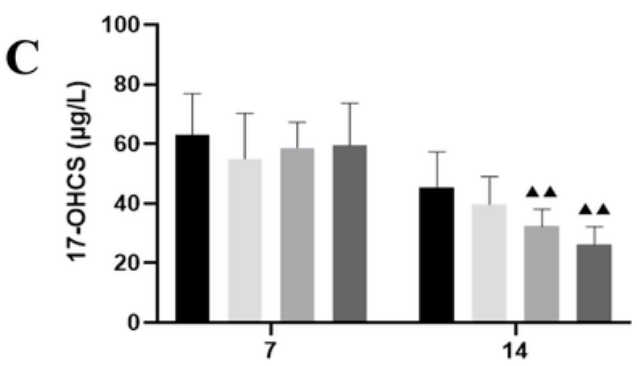

Days/d

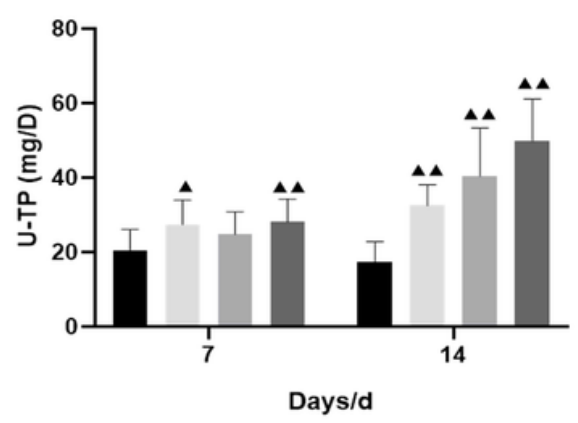

- $\mathrm{CON}$ ADE $150 \mathrm{mg} / \mathrm{kg}$
ADE $100 \mathrm{mg} / \mathrm{kg}$
ADE 200mg/kg

\section{Figure 5}

Effect of different doses of adenine on HPA axis function and renal function in rats. (A-C) indices for HPA axis function, including levels of serum ACTH, CORT and urine 17-OHCS in rats. (D-F) indices for renal function, including levels of blood urea nitrogen (BUN), serum creatinine (Scr), 24-hour urine protein excretion (U-TP/24h) in rats. $n=10$. Small horizontal bars indicate the mean \pm s.d. $\Delta P<0.05, \Delta \Delta P<0.01$ compared with the CON group. 
A

H\&E

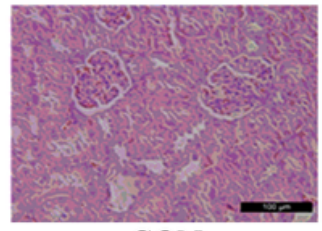

$\mathrm{CON}$

B

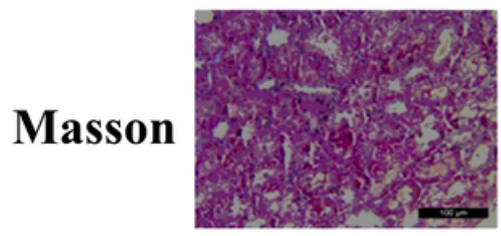

$\mathrm{CON}$

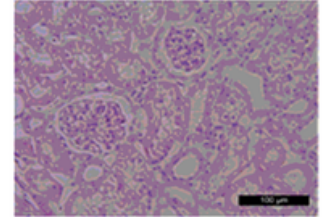

ADE100mg $/ \mathrm{kg}$

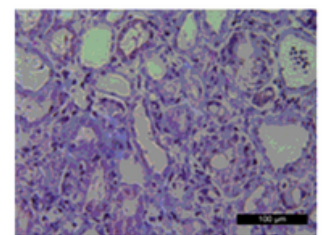

ADE100mg/kg

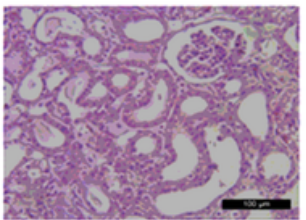

$\mathrm{ADE} 150 \mathrm{mg} / \mathrm{kg}$

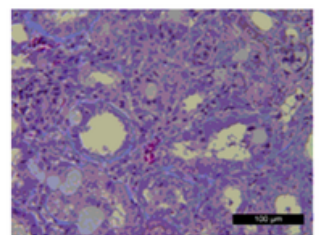

ADE $150 \mathrm{mg} / \mathrm{kg}$

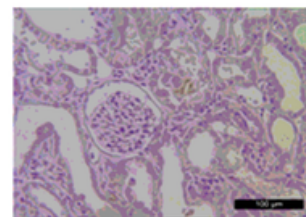

ADE $200 \mathrm{mg} / \mathrm{kg}$

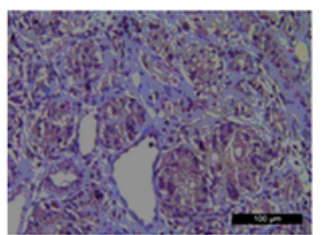

ADE $200 \mathrm{mg} / \mathrm{kg}$

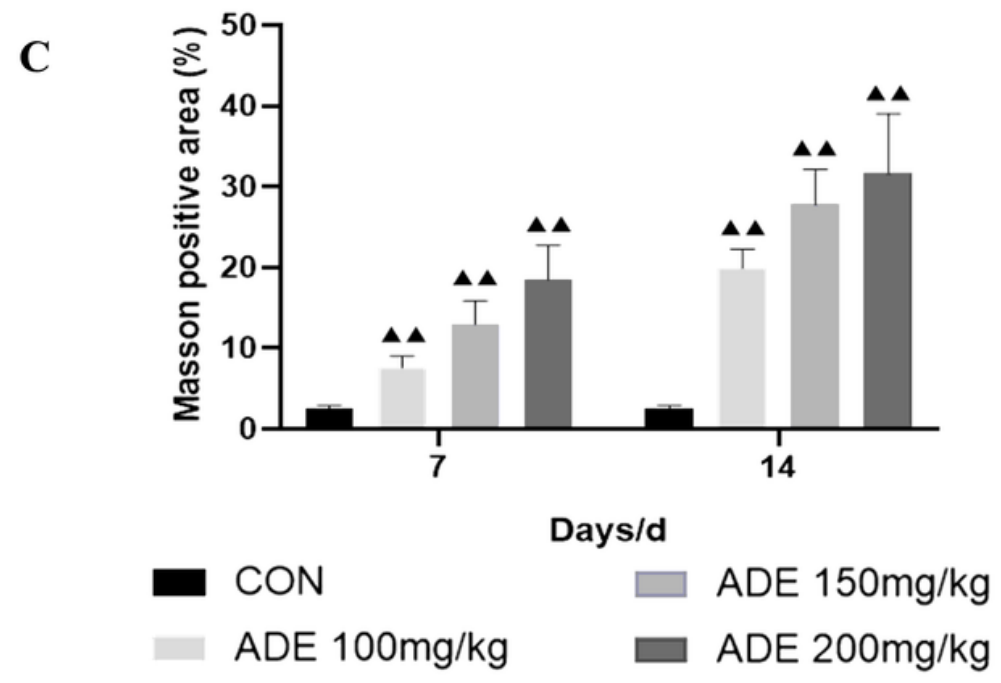

Figure 6

Effect of different doses of adenine on renal pathological change. (A) Respective images of HE stained kidney tissue in rats. (B) Respective images of Masson stained kidney tissue in rats. (C) The degree of Masson positive area (fibrosis) measured by thresholding the percentage area of blue color per field. $n=10$. Small horizontal bars indicate the mean \pm s.d. $\Delta P<0.05, \Delta \mathbf{\Delta} P<0.01$ compared with the CON group. 
A

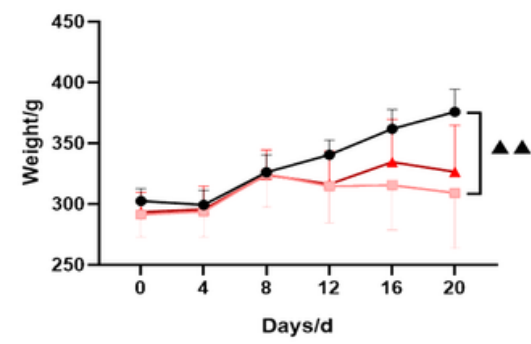

D

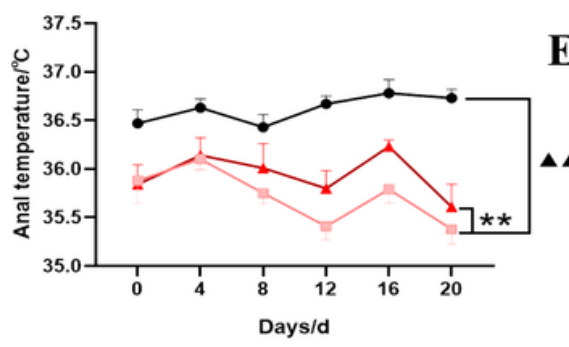

G

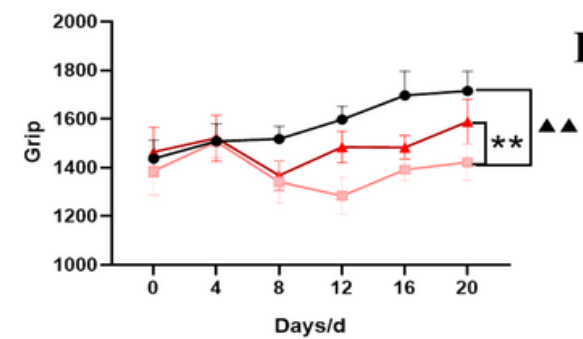

$\rightarrow \mathrm{CON}$

-- ADE $100 \mathrm{mg} / \mathrm{kg}$

$\leftarrow$ ADE $100 \mathrm{mg} / \mathrm{kg}+\mathrm{SQW}$
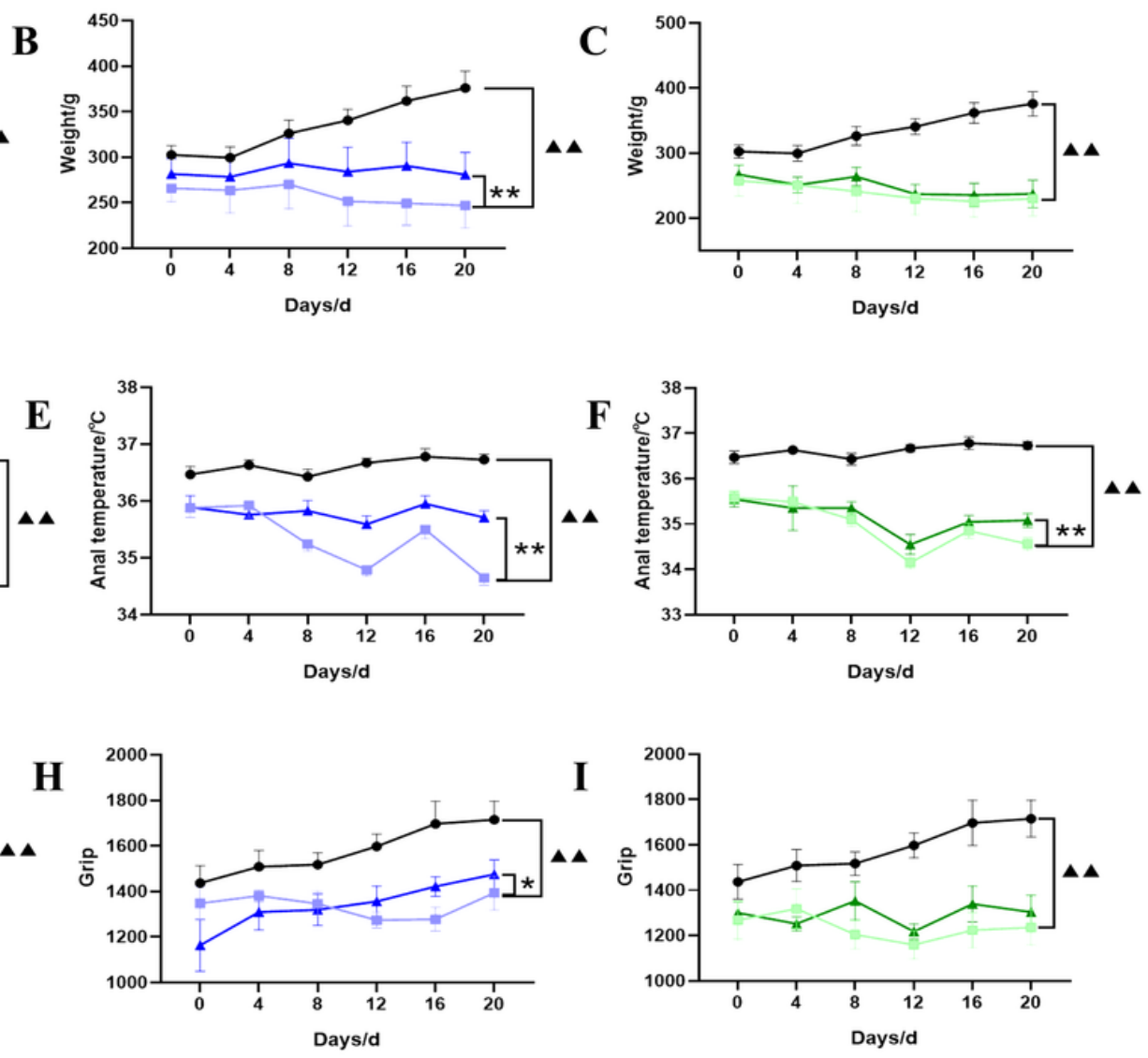

ADE 150mg/kg

ADE $150 \mathrm{mg} / \mathrm{kg}+\mathrm{SQW}$
ADE $200 \mathrm{mg} / \mathrm{kg}$

ADE 200mg/kg+SQW

\section{Figure 7}

Recovery effect of SQW on body weight, anal temperature, grip in KYDS rats induced by the different dose of adenine. (A) effect of $100 \mathrm{mg} / \mathrm{kg}$ SQW on body weight in different doses of adenine induced KYDS rats. (B) effect of $150 \mathrm{mg} / \mathrm{kg} \mathrm{SQW}$ on body weight in different doses of adenine induced KYDS rats. (C) effect of $200 \mathrm{mg} / \mathrm{kg}$ SQW on body weight in different doses of adenine induced KYDS rats. (D) effect of $100 \mathrm{mg} / \mathrm{kg}$ SQW on anal temperature in different doses of adenine induced KYDS rats. (D) effect of 150 $\mathrm{mg} / \mathrm{kg} \mathrm{SQW}$ on anal temperature in different doses of adenine induced KYDS rats. (F) effect of 200 $\mathrm{mg} / \mathrm{kg} \mathrm{SQW}$ on anal temperature in different doses of adenine induced KYDS rats. (G) effect of 100 $\mathrm{mg} / \mathrm{kg}$ SQW on the grip in different doses of adenine induced KYDS rats. (H) effect of $150 \mathrm{mg} / \mathrm{kg} \mathrm{SQW}$ on the grip in different doses of adenine induced KYDS rats. (I) effect of $200 \mathrm{mg} / \mathrm{kg} \mathrm{SQW}$ on the grip in different doses of adenine induced KYDS rats. $n=10$. Small horizontal bars indicate the mean \pm s. $d$.

$\Delta P<0.05, \Delta \Delta \mathrm{A}<0.01$ compared with the CON group; ${ }^{*}<<0.05$, ${ }^{*} P<0.01$ compared with the ADE group. 

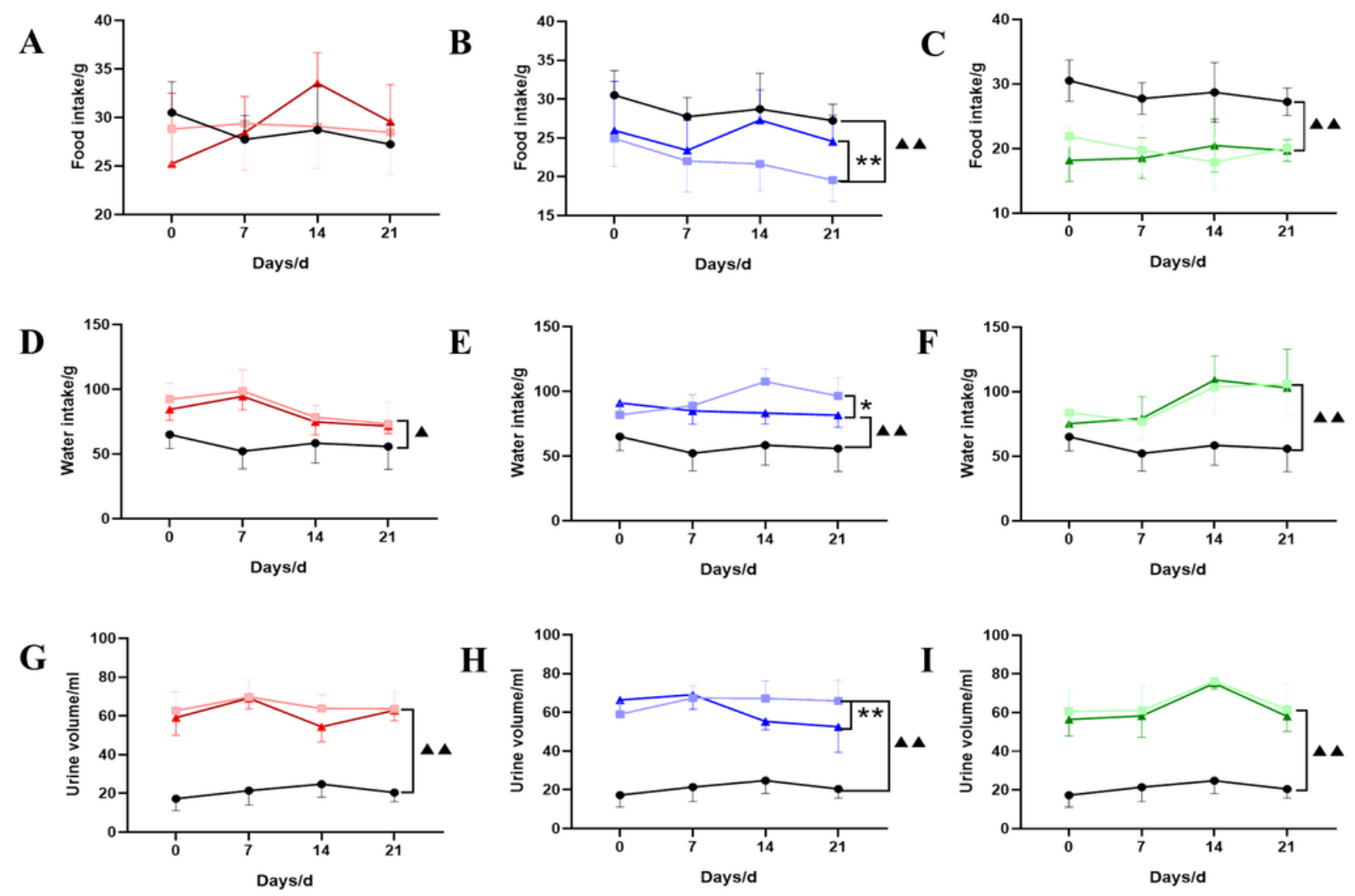

$\rightarrow$ CON

- ADE $100 \mathrm{mg} / \mathrm{kg}$

$\mathrm{ADE} 150 \mathrm{mg} / \mathrm{kg}$

ADE $200 \mathrm{mg} / \mathrm{kg}$

- ADE $100 \mathrm{mg} / \mathrm{kg}+\mathrm{SQW}$

$\mathrm{ADE} 150 \mathrm{mg} / \mathrm{kg}+\mathrm{SQW}$

\section{Figure 8}

Recovery effect of SQW on food intake, water intake and 24h urine volume in KYDS rats induced by the different doses of adenine. (A) effect of $100 \mathrm{mg} / \mathrm{kg} \mathrm{SQW}$ on food intake in different doses of adenine induced KYDS rats. (B) effect of $150 \mathrm{mg} / \mathrm{kg} \mathrm{SQW}$ on food intake in different doses of adenine induced KYDS rats. (C) effect of $200 \mathrm{mg} / \mathrm{kg} \mathrm{SQW}$ on food intake in different doses of adenine induced KYDS rats. (D) effect of $100 \mathrm{mg} / \mathrm{kg} \mathrm{SQW}$ on water intake in different doses of adenine induced KYDS rats. (E) effect of $150 \mathrm{mg} / \mathrm{kg}$ SQW on water intake in different doses of adenine induced KYDS rats. (F) effect of 200 $\mathrm{mg} / \mathrm{kg}$ SQW on water intake in different doses of adenine induced KYDS rats. (G) effect of $100 \mathrm{mg} / \mathrm{kg}$ SQW on urine volume in different doses of adenine induced KYDS rats. $(\mathrm{H})$ effect of $150 \mathrm{mg} / \mathrm{kg}$ SQW on urine volume in different doses of adenine induced KYDS rats. (I) effect of $200 \mathrm{mg} / \mathrm{kg} \mathrm{SQW}$ on urine volume in different doses of adenine induced KYDS rats. $n=10$. Small horizontal bars indicate the mean \pm s.d. $\Delta P<0.05, \Delta \Delta P<0.01$ compared with the CON group; ${ }^{*}<<0.05, * * P<0.01$ compared with the ADE group. 

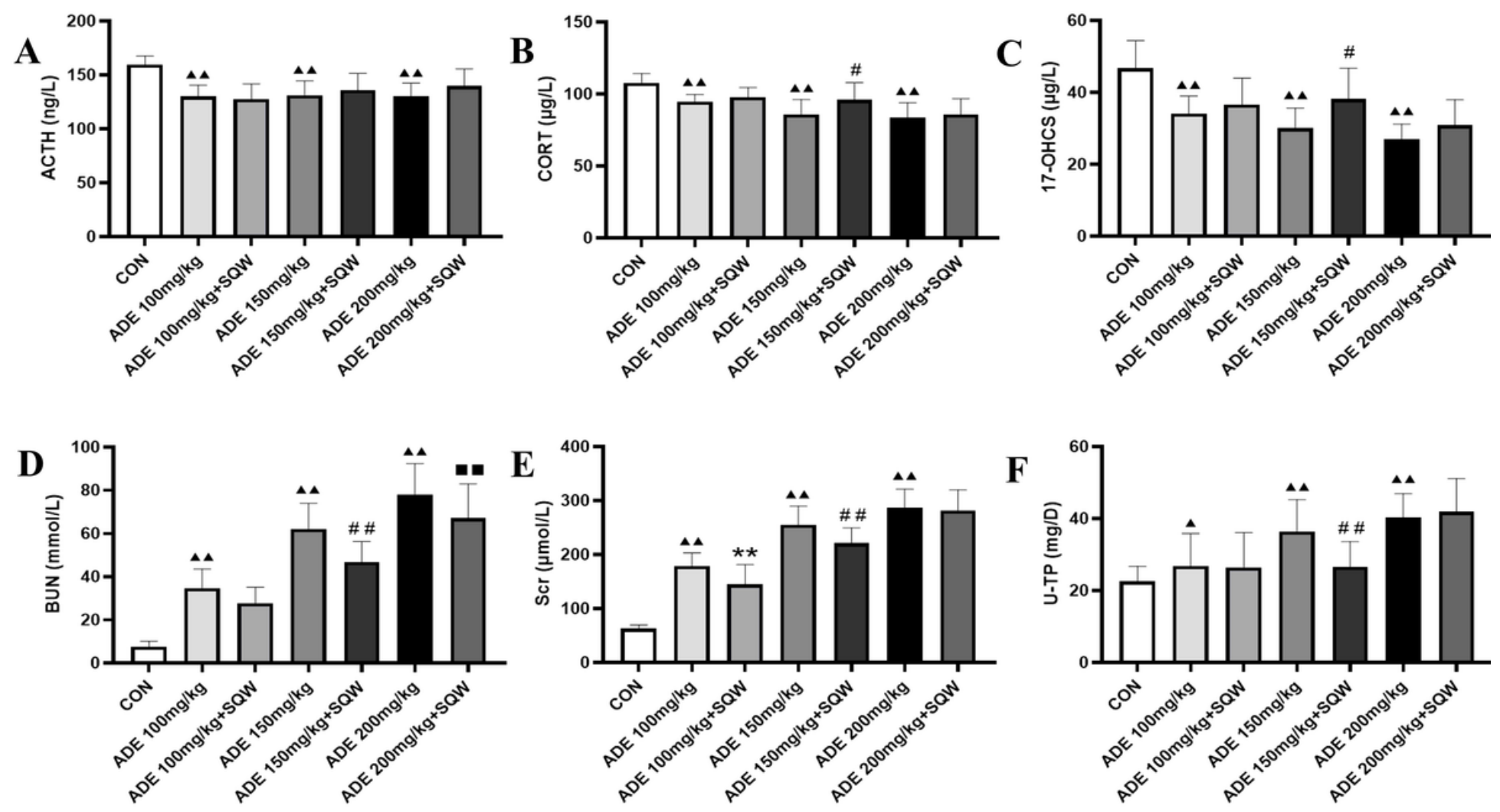

Figure 9

Recovery effect of SQW on HPA axis function and renal function in rats. (A-C) indices for HPA axis function in KYDS rats induced by the different doses of adenine, including levels of serum ACTH, CORT and urine 17-OHCS. (D-F) indices for renal function, including levels of blood urea nitrogen (BUN), serum creatinine (Scr), 24-hour urine protein excretion (U-TP) in rats. $n=10$. Small horizontal bars indicate the mean \pm s.d. $\Delta P<0.05, \Delta \Delta P<0.01$ compared with CON group; ${ }^{*} P<0.05,{ }^{*} \mathrm{P}<0.01$ compared with $A D E$ $100 \mathrm{mg} / \mathrm{kg}$ group. $\# \mathrm{P}<0.05$, \# \#P<0.01 compared with $A D E 150 \mathrm{mg} / \mathrm{kg}$ group; $\mathrm{P}<0.05, \square \square P<0.01$ compared with ADE $200 \mathrm{mg} / \mathrm{kg}$ group. 
A

H\&E
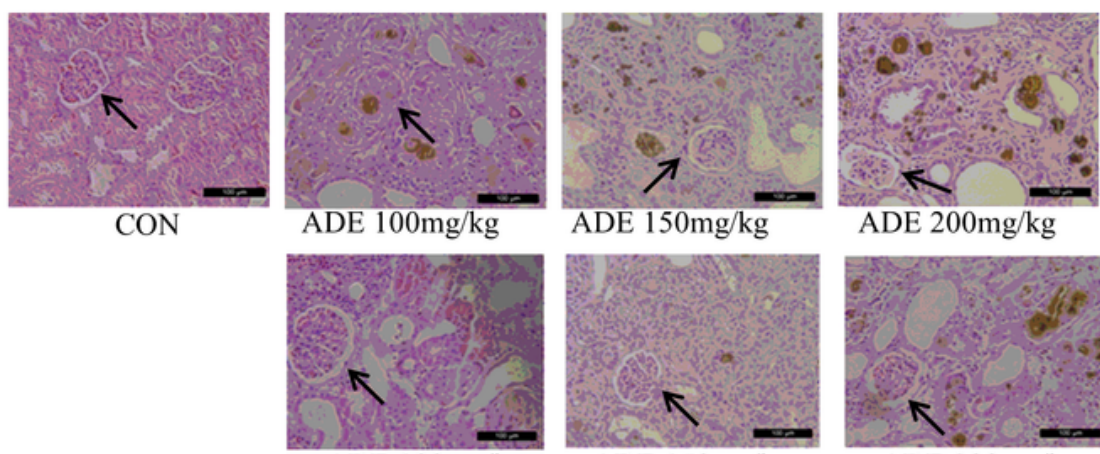

ADE $100 \mathrm{mg} / \mathrm{kg}$

+ SQW

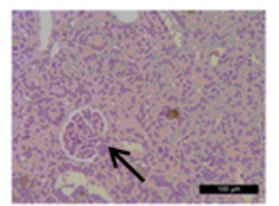

ADE $150 \mathrm{mg} / \mathrm{kg}$

+ SQW

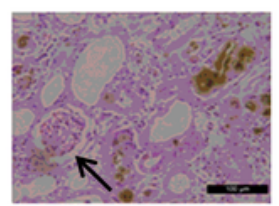

ADE $200 \mathrm{mg} / \mathrm{kg}$

+ SQW

B

Masson
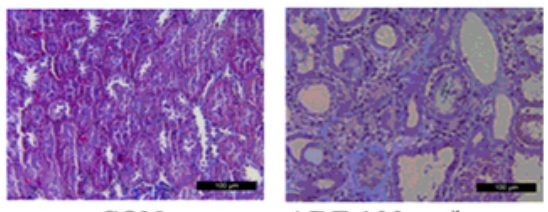

ADE $100 \mathrm{mg} / \mathrm{kg}$

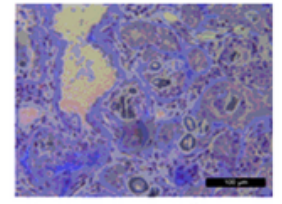

ADE $150 \mathrm{mg} / \mathrm{kg}$
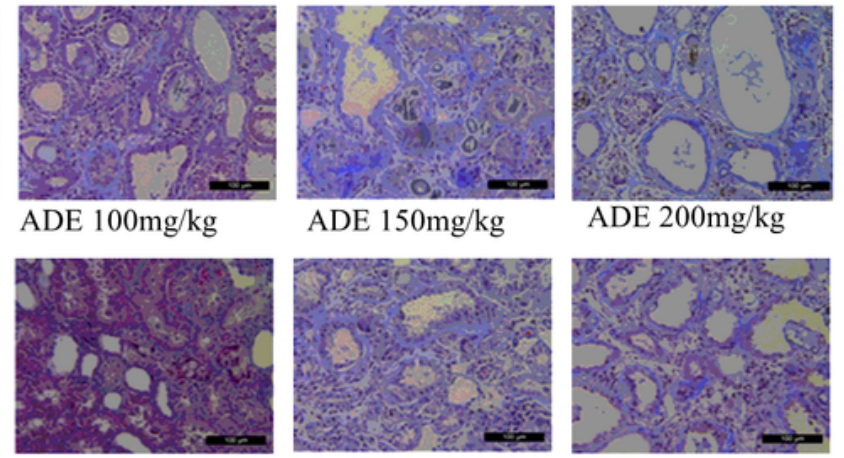

ADE $100 \mathrm{mg} / \mathrm{kg}$

+ SQW

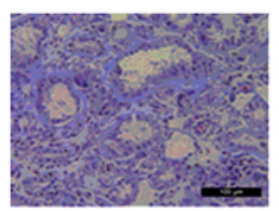

ADE $150 \mathrm{mg} / \mathrm{kg}$

+ SQW

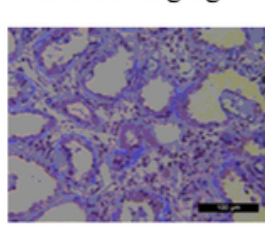

ADE $200 \mathrm{mg} / \mathrm{kg}$

+ SQW

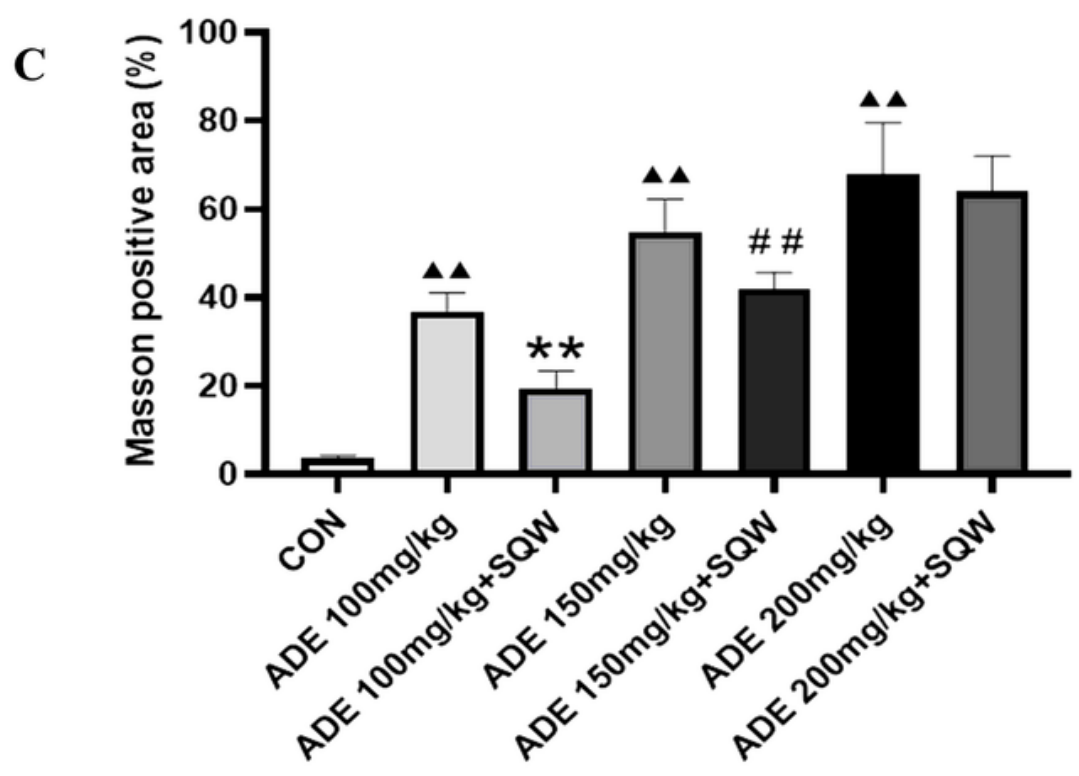

Figure 10

Recovery effect of SQW on renal pathological change in KYDS rats induced by the different doses of adenine. (A) Respective images of HE stained kidney tissue in rats. (B) Respective images of Masson stained kidney tissue in rats. (C) The degree of Masson positive area (fibrosis) measured by thresholding the percentage area of blue color per field. $n=10$. Small horizontal bars indicate the mean \pm s.d. $\Delta P<0.05$, $\Delta \Delta P<0.01$ compared with CON group; ${ }^{*} P<0.05$, ${ }^{\star *} P<0.01$ compared with $A D E 100 \mathrm{mg} / \mathrm{kg}$ group. $\# P<0.05$, 
\# \#P<0.01 compared with ADE $150 \mathrm{mg} / \mathrm{kg}$ group; $\mathrm{P}<0.05$, — $\mathrm{P}<0.01$ compared with ADE $200 \mathrm{mg} / \mathrm{kg}$ group. 